\title{
Relating Residual Stresses to Machining and Finishing in Silicon Carbide
}

\author{
Benjamin P. Groth ${ }^{\mathrm{a}, \mathrm{b}}$, Sean M. Langan ${ }^{\mathrm{a}}$, Richard A. Haber ${ }^{\mathrm{a}}$ and Adrian B. Mann*a,c \\ ${ }^{a}$ Materials Science and Engineering Department, Rutgers University, 607 Taylor Road, Piscataway, NJ08854, USA. \\ ${ }^{\mathrm{b}}$ Now at: Vesuvius USA, Pittsburgh, Pennsylvania, USA. \\ `Biomedical Engineering Department, Rutgers University, 599 Taylor Road, Piscataway, NJ08854, USA. \\ *Corresponding author, T:1-848-445-8421; F:1-732-445-3258; email: abmann@rci.rutgers.edu
}

\begin{abstract}
Machining and surface finishing is essential in the processing of many ceramics, however, it can be detrimental to subsequent performance as it introduces residual stresses and structural defects. Using micro-Raman spectroscopy the residual stress and crystallinity of hotpressed $\mathrm{SiC}$ tiles was examined after finishing with several different methods. $514 \mathrm{~nm}$ and 633 $\mathrm{nm}$ lasers in both conventional and confocal settings enabled stress as a function of depth to be assessed. Single crystal, electronics grade $\mathrm{SiC}$ was used for comparison. Compressive residual stresses were present at the surface and in the sub-surface region in all the samples, but the surface with lowest roughness and a mirror-like finish had the highest residual stress. Crystallinity in $\mathrm{SiC}$ is reduced by defects and stacking faults produced during machining and finishing. The finer surface finishes had higher residual stresses, but were actually found to have better crystallinity than the rough surfaces. The dependence of residual stress and crystallinity on surface roughness is attributed to a change in the mode of ceramic removal from energetic and brittle for rough finishes to ductile for the shallow cuts of smooth finishes. The dependence of stress and crystallinity on roughness means a balance must be struck between smoothness, crystallinity and stresses in choosing the best finish for a given application.
\end{abstract}

Keywords: A. Finishing; B. Spectroscopy; D. SiC; E. Armor. 


\section{Introduction}

\subsection{Silicon Carbide}

Silicon Carbide is of great importance as a structural ceramic as it is amongst the hardest materials known [1]. As such, machining $\mathrm{SiC}$ parts into the required shape and form for a given application can be difficult and may yield unwanted defects. Whether the pieces are machined by grinding and polishing, laser-assisted techniques, or ultrasonic techniques [2], the potential for residual stresses to form at the surface is always present. It is important to know how much stress machining can impart to components, since this stress may be either detrimental, leading to early failure, or advantageous, by helping to toughen the component.

Silicon carbide's crystal structure consists of tetrahedra of either $4 \mathrm{Si}$ atoms and $1 \mathrm{C}$ atom, or $4 \mathrm{C}$ atoms and one $\mathrm{Si}$ atom [3]. The tetrahedra can be stacked in either parallel or antiparallel sequences, and this stacking allows for $\mathrm{SiC}$ to take on many different polytypes. The most common polytypes are a cubic structure known as 3C (or beta-SiC) and 2 hexagonal phases $4 \mathrm{H}$ and $6 \mathrm{H}$, known as alpha-SiC. Each polytype has slightly different mechanical, optical and electronic properties that make them useful for specific applications for instance as armor ceramics, power electronic semiconductors or industrial abrasives [4]. As a result of their different structures each polytype has its own unique Raman spectrum. This makes Raman spectroscopy very useful for characterizing variations in polytype within a sample and, significantly for the current study, changes in the Raman peak position can be related to strains (and hence stresses) in the $\mathrm{SiC}[5,6]$.

In single crystal $\mathrm{SiC}$, stress concentrators take the form of micropipes $[7,8]$ and dislocations [8]. For some applications what is more detrimental than the increased stress is the 
fact that these features often impinge on electronic properties and therefore performance when used as single crystal semiconductors. As electronic properties are less important for bulk polycrystalline samples used in structural applications this effect is not so significant, nonetheless when machining $\mathrm{SiC}$ it is important to limit the defects so they are not able to act as stress intensifiers. In bulk, polycrystalline samples, stress points can come from processing inclusions and thermal stresses $[9,10]$ created during hot-pressing or heat treating. If the density of defects is large enough, different polytypes with mis-matching lattices are formed or stacking faults are present it can also cause a significant stress in the sample [11,12]. Machining can further augment these stresses by introducing more stacking faults and increasing the defect density.

\subsection{Raman Spectroscopy:}

Raman spectroscopy is a form of vibrational spectroscopy that relies on inelastic scattering of monochromatic light to provide vibrational, rotational and other low-frequency mode information about a material system [13]. Data obtained from Raman spectroscopy comes in the form of a vibrational spectra. For a given material system, this spectrum will be unique, and contain information related to bonding between atoms within that system. The theory and fundamentals of Raman have been discussed at great length elsewhere [13-15], as well as the ability to use Raman as a stress measuring tool [16-21]. In the case of silicon carbide the numerous polytypes that can form all have unique Raman spectra. This allows for easy characterization and distinction between polytypes, as seen in Figure 1. The use of Raman to measure stress in silicon carbide has been examined quite thoroughly. However, these experiments have been done mostly on the cubic, 3C, polytype [12,22-28] because of its importance and wide usage in the power electronics field. Only a few papers $[5,6,29]$ discuss the 
use of Raman for stress analysis in $6 \mathrm{H}-\mathrm{SiC}$. In the current study we have focused on the equations developed by Liu and Vohra [5,6] for analysis of data as these are widely accepted for use on $6 \mathrm{H}$ silicon carbide.

The micro-Raman system used in the study was an inVia ${ }^{\mathrm{TM}}$ from Renishaw Inc (New Mills, Wotton-under-Edge Gloucestershire, GL12 8JR, UK) with $633 \mathrm{~nm}$ and $514 \mathrm{~nm}$ lasers. The instrument's resolution in terms of deconvoluting overlapping peaks is on the order of 5 wavenumbers with a precision of $0.05 \mathrm{~cm}^{-1}$, and an accuracy of $0.25 \mathrm{~cm}^{-1}$. This means that peaks closer than $5 \mathrm{~cm}^{-1}$ could not be deconvoluted, however for the peaks of interest in silicon carbide this is not an issue. The penetration of the laser into the sample and hence the depth from which information is obtained is wavelength dependent and a function of the material being investigated. Penetration depth, $D_{p}$, can be estimated from the absorption coefficient, $\alpha[19]$ :

$$
D_{p}=\frac{1}{\alpha(\lambda)}
$$

where $\alpha$ depends on the wavelength, $\lambda$. In the case of $\mathrm{SiC}$ the $514 \mathrm{~nm}$ wavelength has a higher absorption coefficient than the $633 \mathrm{~nm}$ wavelength, which allows for analysis at different depths $[30,31]$.

Penetration depth can be further tuned by using confocal Raman spectroscopy [32-36]. This involves the beam being focused with a numerical aperture, and then shone onto the sample. A confocal aperture, which can be a physical pinhole or a "virtual" pinhole, acts like a slit to block the path of some of the reflected beam. This allows only photons from a narrow focal plane to be focused on the detector. For non-confocal Raman spectroscopy the beam interacts with the sample at a range of depths and all of these are detected by the Charge Coupled Device 
(CCD) camera, but with the confocal feature light from only a narrow range of depths below the material's surface is sampled; the confocal aperture excludes light from higher and lower depths. This allows each laser available to the Raman operator to be used for two depths settings: a normal depth setting that goes farther into the sample and a confocal depth setting closer to the sample's surface. By combining multiple lasers in their normal and confocal modes, four depths for each sample have been investigated. These are, in order of increasing penetration: confocal with $514 \mathrm{~nm}$ laser; confocal with $633 \mathrm{~nm}$ laser; non-confocal $514 \mathrm{~nm}$ laser; non-confocal $633 \mathrm{~nm}$ laser. The approximate, respective, penetration depths for each setting are given by the absorption properties of sintered $\mathrm{SiC}[14]$ as, respectively, $1.5 \mu \mathrm{m}, 2 \mu \mathrm{m}, 3 \mu \mathrm{m}$ and $3.5 \mu \mathrm{m}$.

\section{Materials and Methods}

\subsection{Material Preparation}

Four silicon carbide tiles ( $\mathrm{SiC}-\mathrm{N}$ by $\left.\mathrm{Cercom}{ }^{\circledR}\right)$ cut from a larger billet of hot pressed alpha-SiC with $6 \mathrm{H}$ structure and grains of $\approx 4.1 \mu \mathrm{m}$ in size were provided by the Army Research

Laboratory, Aberdeen Proving Grounds, MD for this study. The tiles were several millimeters in thickness and had standard, rotary ground, mirror finish and grit-blast surfaces, respectively, on one side. The standard finish, Figure 2(a), was obtained after a surface grind and light sand blasting of the hot pressed SiC-N tile. The sand blasting removed any carbon on the tile surface left over from the pressing process which uses a graphite sheet to minimize the interaction with the die. The reverse side of the tiles was finished in the same manner as the standard sample, essentially as-pressed with minimal finishing, and the two were optically similar in appearance. To ensure that the initial states for each of the tile surfaces where consistent the reverse side of the tiles was used as a baseline. The expectation was that each of the reverse sides and the standard finish would have the same initial stress. The grit blast samples were impacted with fine 
steel shot which, as it can be seen from the SEM image in Figure 2(b), gives significant deformation on the machined surface of the tile. The rotary ground samples, Figure 2(c), were taken from the as-pressed state to their finished state through the use of diamond grinding wheels, running on a rotary grinder. The mirror polish samples were taken from the as-pressed state to a mirror-like finish through the use of successive polishing with finer and finer diamond abrasives. The mirror finished sample displayed the lowest amount of surface roughness with $\mathrm{R}_{\mathrm{a}}$ $\approx 14 \pm 3 \mathrm{~nm}$ compared to $\mathrm{R}_{\mathrm{a}} \approx 483 \pm 34 \mathrm{~nm}$ for grit blast, $\mathrm{R}_{\mathrm{a}} \approx 264 \pm 41 \mathrm{~nm}$ for rotary ground and $\mathrm{R}_{\mathrm{a}} \approx 236 \pm 25 \mathrm{~nm}$ for standard finish. Single crystal samples for comparison to the tiles of $6 \mathrm{H}-\mathrm{SiC}$ (0001) were obtained from MTI Corporation, Richmond, CA 94804, USA. These had roughness of $\mathrm{R}_{\mathrm{a}}<1 \mathrm{~nm}$ and the Raman data suggested that they were essentially stress-free at the depths being studied during the testing.

In order to evaluate and quantify the stress in each sample due to machining Raman maps were created from measurements obtained at points spaced $2 \mu \mathrm{m}$ apart over $40 \mu \mathrm{m} \times 40 \mu \mathrm{m}$ areas. This size was set based on the capabilities of the Raman system and to avoid spatial overlap of the Raman signal. The Raman system was equipped with an 1800 1/mm diffraction grating for both the Ar ion $514 \mathrm{~nm}$ laser and the HeNe $633 \mathrm{~nm}$ laser. The maps acquired were point maps where at each point a full spectrum was obtained and not just one peak as examined in a line focus map. Maps were acquired on several different areas of each sample's surface and stress values were averaged over all maps. Sample maps can be seen in the results section which shows the differences between each of the surface finishes and also the variations with depth for a given finish. Along with the machined surfaces, maps were created of the tiles' reverse (unmachined) sides.

\subsection{Micro-Raman Spectroscopy}


Variations in stress with depth were examined using the micro-Raman's confocal settings along with the two different excitation wavelengths. The confocal setting enables the Raman spectra to be obtained at a shallower depth into the sample than with the normal setting. It uses a virtual pinhole, created by slits in the spectrometer path and a decreased CCD scan area, as opposed to a physical pinhole in the laser path or in the microscope. The slits spacing is decreased from $50 \mu \mathrm{m}$ in the regular setup, to $20 \mu \mathrm{m}$ in the confocal, in order to limit the angle of returning light that is analyzed. Similarly, the CCD area is taken from 15 pixels down to only 3 pixels; this allows for in-depth spatial resolution at a cost of signal-to-noise ratio. In order to achieve intensity counts similar to those in the regular setting, several scan iterations were performed. With regards to excitation wavelength the absorption coefficient of silicon carbide increases for shorter wavelengths, so the $514 \mathrm{~nm}$ laser penetrates less than the $633 \mathrm{~nm}$ laser. Hence, the different wavelengths enable stress to be examined at different depths.

Averaged stress and peak width values were obtained from all the data in a scan for the specified depth of penetration. To extract the values for each penetration depth the data was weighted based on the penetration of the laser used. For example, the $514 \mathrm{~nm}$ confocal setting has a penetration of $\sim 1.5 \mu \mathrm{m}$ in the polycrystalline $\mathrm{SiC}$ tiles, so for this configuration the stress and peak width values are a weighted average over the first $1.5 \mu \mathrm{m}$ of depth. For the $633 \mathrm{~nm}$ confocal setting the penetration is greater $(\sim 2 \mu \mathrm{m})$ and the values of stress and peak width from 1.5-2 $\mu \mathrm{m}$ depth are obtained by subtracting the values for the $514 \mathrm{~nm}$ confocal setting. The other penetration depth values are found similarly: for the $514 \mathrm{~nm}$ regular setting the values at a penetration depth of $\sim 3 \mu \mathrm{m}$ are found; for the $633 \mathrm{~nm}$ regular setting at a penetration depth of 3.5 $\mu \mathrm{m}$. 


\subsection{Analysis of Raman Data}

The Raman spectral data was processed using the WiRE3.2 software available from Renishaw. Spectrum intensities were normalized to the largest intensity peak, the transverseoptical (TO) peak at $789.2 \mathrm{~cm}^{-1}$, and corrected for baseline. Curve fitting was performed using the software's curve fit feature, with curve parameters set to allow mixed Gaussian-Lorentzian behavior. These parameters were saved and applied to each spectra in the acquired maps, and peak position and width information for the TO peak was extracted for each map point. These values were used to create a contour plot of peak shift and an average value for the peak width over the mapped area. Peak shift values were then turned into stresses using:

$$
v_{T O}=3.11 P+789.2
$$

where $\mathrm{P}$ is applied pressure, $v_{T O}$ is the measured TO peak position, and the value 789.2 is the unstressed peak position for the TO peak in $\mathrm{cm}^{-1}$. This is a modified version of the equation developed by Liu and Vohra [5,6]. They investigated ultrahigh pressures where a quadratic term is needed; this term is negligible for the stresses seen in the current study. The use of this equation to relate stress to shift in a Raman peak assumes that the samples have no preferred crystal orientation; this was confirmed by X-ray diffraction (XRD) studies of the tiles which showed no evidence of texture. A Bruker AXS Inc., WI 53711-5373, USA, HiStar ${ }^{\mathrm{TM}}$ system with a $2 \mathrm{D}$ array detector, and $\mathrm{Cu}$ radiation with a $0.05 \mathrm{~mm}$ diameter collimator was used for the XRD studies.

\section{Results}

\subsection{Single Crystal}

Raman maps taken over the unstressed single crystal silicon carbide showed no significant peak shift which is consistent with it being unstressed at the depths of interest. The 
peak position values obtained were statistically close to the accepted average value of $789.2 \mathrm{~cm}^{-}$

${ }^{1}$, for the TO peak [5], having deviations less than $0.05 \mathrm{~cm}^{-1}$ from the average over the mapped section. The penetration depth is large for single crystal $6 \mathrm{H}-\mathrm{SiC}$ and on the order of $10 \mu \mathrm{m}$ based on absorption coefficients from Werheit and Schwetz [14]. The spectra acquired at each map point are weighted averages from the surface to the specified depth in each sample. Peak width values were also collected and averaged over the map area to provide a baseline peak width for comparison.

\subsection{Machined Samples}

Peak shifts due to machining are typically to higher wavenumbers, as shown by the example of Figure 3, which corresponds to compressive stress. However, relatively small tensile stress regions are occasionally seen in localized areas. A stress value was found for each of the four surface finishes by averaging over all the data points on all of the Raman maps collected. These average were always relatively large and compressive. For the minimum penetration depth (confocal setting with $514 \mathrm{~nm}$ wavelength) the average stresses were: $-220 \pm 25 \mathrm{MPa}$ for the standard finish; $-371 \pm 27 \mathrm{MPa}$ for the grit blast sample; $-485 \pm 33 \mathrm{MPa}$ for the rotary finish; $601 \pm 38 \mathrm{MPa}$ for the mirror finish. Raman maps for all 4 samples at each setting $(514 / 633 \mathrm{~nm}$ wavelength, confocal/normal setting) are shown in Figures 4, 5, 6 and 7. In comparison, the Raman data for the reverse sides of all tiles showed a very uniform average stress of $-208 \pm 21$ MPa with little variation with depth. Within the margin of error this was the same compressive stress as that seen for the sample with the standard finish.

For each of surface finishes there was considerable variation in stress over each map, though it was least for the standard finish. As the penetration depth increased there was a marked reduction in the range of the stresses measured and also in the average stress over the mapped 
area. For all of the surfaces except the standard the average stress value dropped by $25-35 \%$ between the shallowest confocal setting and the deepest regular setting. This indicates that the machining stresses decrease with depth from the surface. This stress gradient is greatest in the mirror finished sample, but is practically non-existent in the standard finish sample. Figure 8 shows the average stress value of each sample at each of the four depth settings. Also plotted on Figure 8 is the average stress over the surface for the single crystal $\mathrm{SiC}$ (stress $\approx 0 \mathrm{MPa}$ ). The standard sample and the reverse sides show a consistent stress level with depth (only the standard sample is shown on the Figures as the reverse sides were essentially the same).

Analyzing the TO peak width (which is related to crystallinity) showed that the samples split into 2 groups: the grit blast and rotary finish showed similar and higher average relative width values of $1.8 \mathrm{~cm}^{-1}$ for the shallowest depths (514 nm wavelength and confocal setting); the standard and mirror finish samples showed peak widths of $1.5 \mathrm{~cm}^{-1}$ for the shallowest depths. These values were normalized with respect to the average width values of the single crystal SiC, at the respective depth settings. The width values for all of the samples at the different laser and confocal settings can be seen in Figure 9. All of the samples tend towards a single relative peak width value as the penetration depth increases. A sample Raman spectrum showing the variation in width, as well as peak position can be seen in Figure 10.

\section{Discussion}

The machined stress values in each sample show a linear trend towards a baseline residual stress value, which is that of the unmachined, reverse sides. The intercept of the trendline with the baseline residual stress value gives an approximate maximum depth at which the machining process has affected the sample. For the higher stressed mirror finish and rotary ground samples, this value is $6.6 \pm 0.5 \mu \mathrm{m}$ and $6.6 \pm 0.7 \mu \mathrm{m}$, respectively. In the grit blast sample, 
this value was lower, at $4.4 \pm 0.4 \mu \mathrm{m}$, indicating that the deformation and stress concentration was much nearer to the surface. In shot peened or grit blasted samples the deformation generally tends to be nearer the surface as the intent is to apply residual compressive surface stresses from densification in the impacted regions. In single crystal samples it should be noted that given the very low absorption coefficient of $6 \mathrm{H}-\mathrm{SiC}$, and the wavelength dependence of this coefficient, there was no wavelength that could be used to analyze the shallowest depths with Raman spectroscopy. There are z-scan techniques that utilize through-focus scanning to get depth resolution, but the depth estimation falls off quickly even with small below-focus step sizes [15].

An analysis of the peak width data shows that all for the machined samples the trend with increasing penetration depth is towards the baseline width value of the unmachined side. There is a relationship between stress and peak width $[5,6,38]$, but in this case the differences in peak width data and stress between the samples do not closely follow each other. Specifically, the mirror finish has less effect on peak width, and therefore crystallinity, than it does on residual stress. Grit blast and rotary ground, however, both increase the peak width considerably, but give less residual stress than the mirror finish; substantially less for the grit blast sample. Thus, it could be misleading to just consider residual stress as an indicator of surface and sub-surface damage and, likewise, it could be misleading to just consider changes in peak width. For a given application either or both could be detrimental to the ultimate performance of the SiC-N.

$\mathrm{SiC}$ is anisotropic and for the machined samples studied the stresses introduced are likely to be anisotropic and bi-axial (or possibly even tri-axial). However, the tiles tested consist of randomly-oriented polycrystalline $\mathrm{SiC}$, this eliminates many of the effects on peak shifts due to anisotropy. Consequently, the peak shifts are close to those that would be seen under hydrostatic stress, and any difference in the magnitude of stresses with direction will be relatively small 
compared to the overall shifts in the Raman peaks. As a result using equations for isotropic samples under hydrostatic pressure to obtain stress measurements from Raman is valid for the four surface finishes studied. In the case of the single crystal sample the material is truly anisotropic elastically, however the Raman measurements shows no sign of a peak shift in the pristine surface so the method of quantifying stress does not seem to be an issue in this case. Though it was not used in the current study a polarized analysis of the Raman spectra would have given more information on any anisotropy present. Overall, issues related to anisotropy in the samples might be expected to introduce a small error in the stress per wavenumber shift $\left(\mathrm{GPa} / \mathrm{cm}^{-1}\right)$ when using the purely hydrostatic stress model. However, the comparative data, specifically between different machining methods, and the data with respect to changes in stress with depth remain valid for the samples studied.

There are limits to the ability of micro-Raman as used in this study to characterize the stress state, in particular whether hydrostatic, biaxial or triaxial stresses are present. This does not distract from the significance of the results in showing that surface damage from machining extends many microns below the surface in SiC-N tiles, and the extent of the damage is a function of the machining methods used. Specifically, the data shows that there is a tendency for finer surface finishes to result in greater compressive sub-surface residual stresses, but to give less crystalline disorder when compared to coarser and more energetic surface finishing methods, such as rotary grinding and grit blasting.

\section{Conclusions}

The combination of pressure and shear stress inherent in all machining processes introduces large, compressive residual stresses that persist to a depth of $3.5 \mu \mathrm{m}$ or more. The type of machining and finish (grit blast, rotary or mirror finish) was found to affect the 
magnitude of the average stress at all the depths examined. In this respect the grit blast caused the lowest total increase in stress at all depths, while the mirror finish created the largest stress at all depths, with the rotary finish lying between them. For all the machined samples the average compressive stress was found to be noticeably higher in the first $2 \mu \mathrm{m}$ below the surface when compared to greater depths. In the case of the mirror finished sample the first $2 \mu \mathrm{m}$ exhibited extremely high compressive stresses averaging over $600 \mathrm{MPa}$, with localized peaks close to 1000 MPa. For all the samples there was considerable variation in the magnitude of the stresses over the surface, as shown by the stress maps. This scatter was found to be greater at the shallowest depths examined for all the finishes. The mirror finish gives the most pronounced increase in compressive stress and this is taken to be a result of polishing with fine abrasives.

Silicon carbide $6 \mathrm{H}$ has a strong transverse optical peak at $789.2 \mathrm{~cm}^{-1}$ the width of which can be used as a measure of "crystallinity" or disorder in the crystal structure. This disorder was found to increase with machining, with the increase most pronounced close to the surface. Intriguingly the effect on crystallinity was less pronounced for the mirror finish than for the rotary grind and grit blast surface. Thus, the fine polishing associated with producing a mirror finish gives rise to larger residual stresses, but less disorder in the crystals when compared to the coarser finishes. Fracture mechanics predicts that for finer abrasives with lower depths of removal ductile deformation dominates [39]. The observed differences in residual stress and crystallinity can be explained by, firstly, the transition from brittle deformation for coarser surface finishes to ductile deformation for finer surface finishes and, secondly, the tendency of more energetic surface finishing methods (rotary grind and grit blast) to introduce defects such as stacking faults in $\mathrm{SiC}$. 
Depending on the specific application of the ceramic the changes in crystallinity indicated by the increased peak width may ultimately be as significant in determining the sample's performance as the residual stresses generated by the different machining methods. Thus, the method chosen for finishing the surface should be selected based not only on the roughness required, but also the residual stress and crystallinity that is needed to avoid premature failure.

\section{Acknowledgements}

This material is based upon work supported by the National Science Foundation under Grant No. 1034979. This partially supports the Ceramic, Composite and Optical Materials Center (CCOMC), an NSF I/UCRC, at Clemson University and Rutgers University. Any opinions, findings, and conclusions or recommendations expressed in this material are those of the author(s) and do not necessarily reflect the views of the National Science Foundation. 


\section{References}

1. K.A. Schwetz, Silicon Carbide Based Hard Materials, in: R. Riedel (ed), Handbook of Ceramic Hard Materials, Wiley-VCH Verlag GmbH, Weinheim, Germany, 2008, pp. 683-748.

2. I.D.Marinescu, Handbook of Advanced Ceramic Machining, Taylor \& Francis Group, Boca Raton, 2007.

3. D. Lundquist, On the Crystal Structure of Silicon Carbide and its Content of Impurities, Acta Chem. Scand. 2 (1948) 177-191.

4. S. Saddow, A. Agarwal, Advances in Silicon Carbide Processing and Applications, Massachusetts, Artech House, 2004.

5. J. Liu, Y.K. Vohra, Raman modes of $6 \mathrm{H}$ polytype of silicon carbide to ultrahigh pressures: A comparison with silicon and diamond, Phys. Rev. Lett. 72 (26) (1994) 4105-4108.

6. J. Liu, Y.K. Vohra, Raman modes of $6 \mathrm{H}$ polytype of silicon carbide to ultrahigh pressures Reply, Phys. Rev. Lett. 77 (8) (1996) 1661-1661.

7. M. Sasaki, Y. Nishio, S. Nishino, S. Nakashima, H. Harima, Defect Formation Mechanism of Bulk SiC, Mater. Sci. Forum 264-268 (1998) 41-44.

8. M. Tuominen, E. Prieur, R. Yakimova, R.C. Glass, T. Tuomi, E. Janzen. Defect Analysis in Lely-grown 6H SiC, J. Cryst. Growth 165 (3) (1996) 233-244.

9. M. Hundhausen, R. Pusche, J. Rohrl, L. Ley, Characterization of defects in silicon carbide by Raman spectroscopy, Phys. Stat. Sol. B 245 (7) (2008) 1356-1368. 
10. W. Wesch, Silicon carbide: synthesis and processing', Nucl. Instrum. Methods Phys. Res. Sect. B $116(1-4)$ (1996) 305-321.

11. J. Zhu, S.Y. Liu, J.W. Liang, Raman study on residual strains in thin 3C-SiC epitaxial layers grown on Si(001), Thin Solid Films 368 (2) (2000) 307-311.

12. C. Genzel, A. Klaus, I. Denks, H.G. Wulz, Residual stress fields in surface-treated silicon carbide for space industry - comparison of biaxial and triaxial analysis using different X-ray methods, Mater. Sci. Eng. A 390 (1-2) (2005) 376-384.

13. J.R. Ferraro, K. Nakamoto, C.W. Brown, Introductory Raman Spectroscopy (2nd ed.), Academic Press, San Diego, 2003.

14. H. Werheit, K.A. Schwetz,: Comparative optical investigations of sintered and monocrystalline black and green silicon carbide (SiC), J. Solid State Chem. 177 (2) (2004) 580585.

15. J.L. Bruneel, J.C. Lassegues, C. Sourisseau, In-depth analyses by confocal Raman microspectrometry: experimental features and modeling of the refraction effects, J. Raman Spectrosc. 33 (10) (2002) 815-828.

16. B. Dietrich, and K. Dombrowski, Experimental challenges of stress measurements with resonant micro-Raman spectroscopy, J. Raman Spectrosc. 30 (10) (1999) 893-897.

17. P. Colomban, Analysis of Strain and Stress in Ceramic, Polymer and Metal Matrix Composites by Raman Spectroscopy, Adv. Eng. Mater. 4 (8) (2002) 535-542.

18. L.A. Falkovsky, J.M. Bluet J. Camassel, Strain-fluctuation effect on Raman spectra, Phys. Rev. B: Condens. Matter 55 (22) (1997) R14697-14700. 
19. G. Gouadec, P. Colomban, Raman spectroscopy of nanomaterials: How spectra relate to disorder, particle size and mechanical properties, Prog. Cryst. Growth Charact. Mater. 53 (1) (2007) 1-56.

20. E.M. Anastassakis, Morphic effects in lattice dynamics: in G.K. Horton, A.A. Marahudin (eds.), Dynamical Properties of Solids, North Holland Publishing Company, Amsterdam, Netherlands, 1980, pp. 158-373.

21. L. Colombi Ciacchi, G. Gregori, V. Lughi, A. Rossi, V. Sergo, Piezo-spectroscopy: a materials science perspective: in M. Pandalai (ed.), Recent Research Developments in Applied Spectroscopy 2, Research Signpost, Kerala, India, 1999, pp. 243-272.

22. A. Debernardi, C. Ulrich, K. Syassen, M. Cardona, Raman line widths of optical phonons in 3C-SiC under pressure: First-principles calculations and experimental results, Phys. Rev. B: Condens. Matter 59 (10) (1999) 6774-6783.

23. Z. Feng, W. Choyke, J. Powell, Raman determination of layer stresses and strains for heterostructures and its application to the cubic SiC/Si system, J. Appl. Phys. 64 (12) (1988) $6827-6835$.

24. D. Ghosh, G. Subhash, N. Orlovskaya, Measurement of scratch-induced residual stress within $\mathrm{SiC}$ grains in $\mathrm{ZrB} 2-\mathrm{SiC}$ composite using micro-Raman spectroscopy, Acta Mater. 56 (11) (2008) 5345-5354.

25. C.J. Lee, G. Pezzotti, Y. Okui, S. Nishino, Raman microprobe mapping of residual microstresses in 3C-SiC film epitaxial lateral grown on patterned Si(111), Appl. Surf. Sci. 228 (1-4) (2004) 10-16. 
26. D. Olego, M. Cardona, Pressure dependence of Raman phonons of Ge and 3C-SiC, Phys. Rev. B: Condens. Matter 25 (2) (1982) 1151-1160.

27. H. Mukaida, H. Okumura, J. H. Lee, H. Daimon, E. Sakuma, S. Misawa, K. Endo, S. Yoshida, Raman scattering of SiC: Estimation of the internal stress in 3C-SiC on Si, J. Appl. Phys. 62 (1) (1987) 254-257.

28. D. Matsuoka, H. Yamamoto, S. Nishino, N. Hasuike, K. Kisoda, H. Harima, Raman scattering study of stress distribution around dislocation in SiC, Mater. Sci. Forum 600-603 (2009) 337-340.

29. K. Grodecki, A. Wysmolek, R. Stepniewski, J.M. Baranowski, W. Hofman, E. Tymicki, K. Grasza, Raman piezospectroscopy of phonons in bulk 6H-SiC, Acta Phys. Pol. A 116 (5) (2009) 947-949.

30. S. Nakashima, H. Harima, Raman investigation of SiC polytypes, Phys. Status Solidi A 162 (1) (1997) 39-64.

31. S. Nakashima, Raman imaging of semiconductor materials: characterization of static and dynamic properties, J. Phys.: Condens. Matter 16 (2) (2004) S25-S37.

32. N. Everall, Depth profiling with confocal Raman microscopy, part I, Spectrosc. 19 (10) (2004) 22-27.

33. N. Everall, Depth profiling with confocal Raman microscopy, part II, Spectrosc. 19 (11) (2004) 16-27.

34. N. Everall, Confocal Raman microscopy: Performance, pitfalls, and best practice, Appl. Spectrosc. 63 (9) (2009) 245A-262A. 
35. P. Vicente, D. David, J. Camassel, Raman scattering as a probing method of subsurface damage in SiC, Mater. Sci. Eng. B 80 (1-3) (2001) 348-351.

36. D.M. Lipkin, D.R. Clarke, Sample-probe interactions in Spectroscopy: Sampling microscopic property gradients, J. Appl. Phys. 77 (5) (1995) 1855-1863.

37. A.M. Gigler, A.J. Huber, M. Bauer, A. Ziegler, R. Hillenbrand, R.W. Stark, Nanoscale residual stress-field mapping around nanoindents in SiC by IR s-SNOM and confocal Raman microscopy, Opt. Express 17 (25) (2009) 22351-22357.

38. I. DeWolf, Micro-Raman spectroscopy to study local mechanical stress in silicon integrated circuits, Semicond. Sci. Technol. 11 (2) (1996) 139-154.

39. T.G. Bifano, T.A. Dow, R.O. Scattergood, Ductile-regime grinding: a new technology for machining brittle materials, J. Eng. Ind. 113 (2) (1991) 184-189. 


\section{Figure Captions}

Figure 1: Sample spectra for $4 \mathrm{H}-$, and $6 \mathrm{H}-\mathrm{SiC}$. The variation in both position of optical phonons and unique acoustic modes allow for characterizing different polytypes. Peaks are designated by their propagation direction, with TO, TA and LO being transverse optical, transverse acoustical and longitudinal optical, respectively.

Figure 2: SEM images of the machined surfaces for the a) Standard, b) Grit Blast, c) Rotary Ground, and d) Mirror Finish.

Figure 3: Example spectra showing shift in peak position for a machined $\mathrm{SiC}$ sample compared to the ideal unstressed (single crystal) Raman spectrum. The peak shift of one wavenumber corresponds to a residual stress of $\sim 320 \mathrm{MPa}$. In this case it is compressive as the peak shifts to the right.

Figure 4: Stress maps of the standard finish sample at a) 514nm confocal, b) $633 \mathrm{~nm}$ confocal, c) $514 \mathrm{~nm}$ regular and d) $633 \mathrm{~nm}$ regular settings. The same area is shown in each image.

Figure 5: Stress maps of the grit blast sample at a) 514nm confocal, b) 633nm confocal, c) $514 \mathrm{~nm}$ regular and d) $633 \mathrm{~nm}$ regular settings. The same area is shown in each image.

Figure 6: Stress maps of the rotary ground sample at a) 514nm confocal, b) 633 confocal, c) 514 regular and d) 633 regular settings. The same area is shown in each image.

Figure 7: Stress maps of the mirror finish sample at a) $514 \mathrm{~nm}$ confocal, b) 633 confocal, c) 514 regular and d) 633 regular settings. The same area is shown in each image.

Figure 8: Plot of residual stress values in all samples as they vary with depth. 
Figure 9: Plot of relative peak widths for all samples as they vary with depth.

Figure 10: Typical spectra showing peak position and peak width variations seen. The peaks located at $\sim 768 \mathrm{~cm}^{-1}$ are mostly invariant, while the TO peak at $789.2 \mathrm{~cm}^{-1}$ can both broaden and shift. 
Figure 1

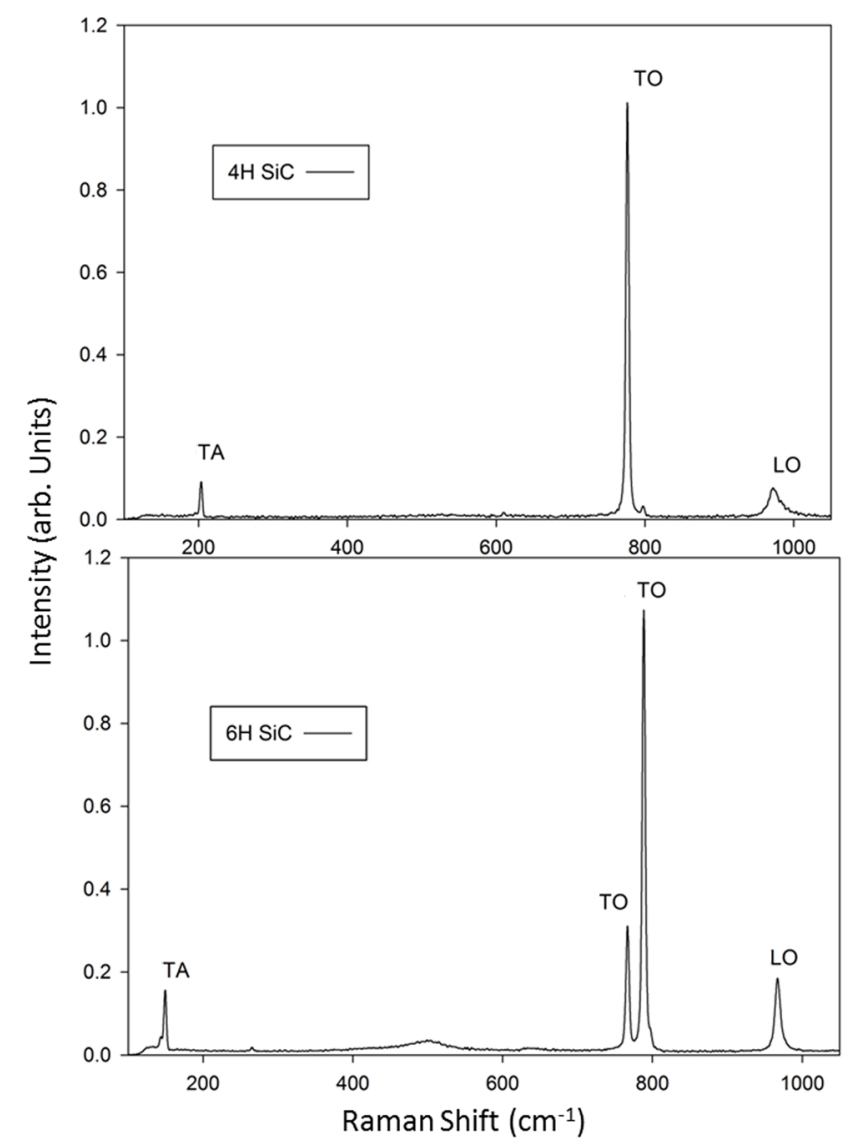


Figure 2

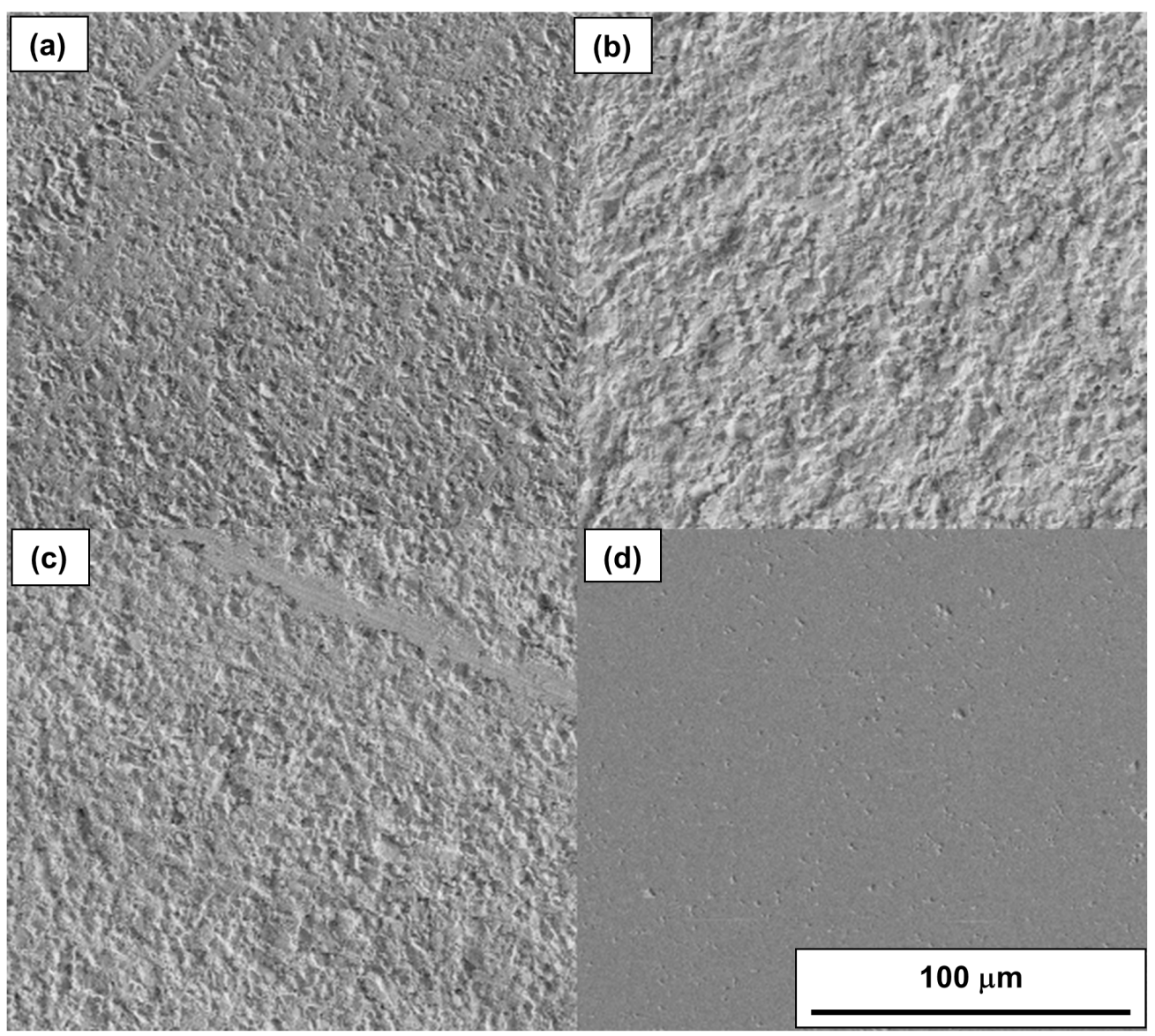


Figure 3

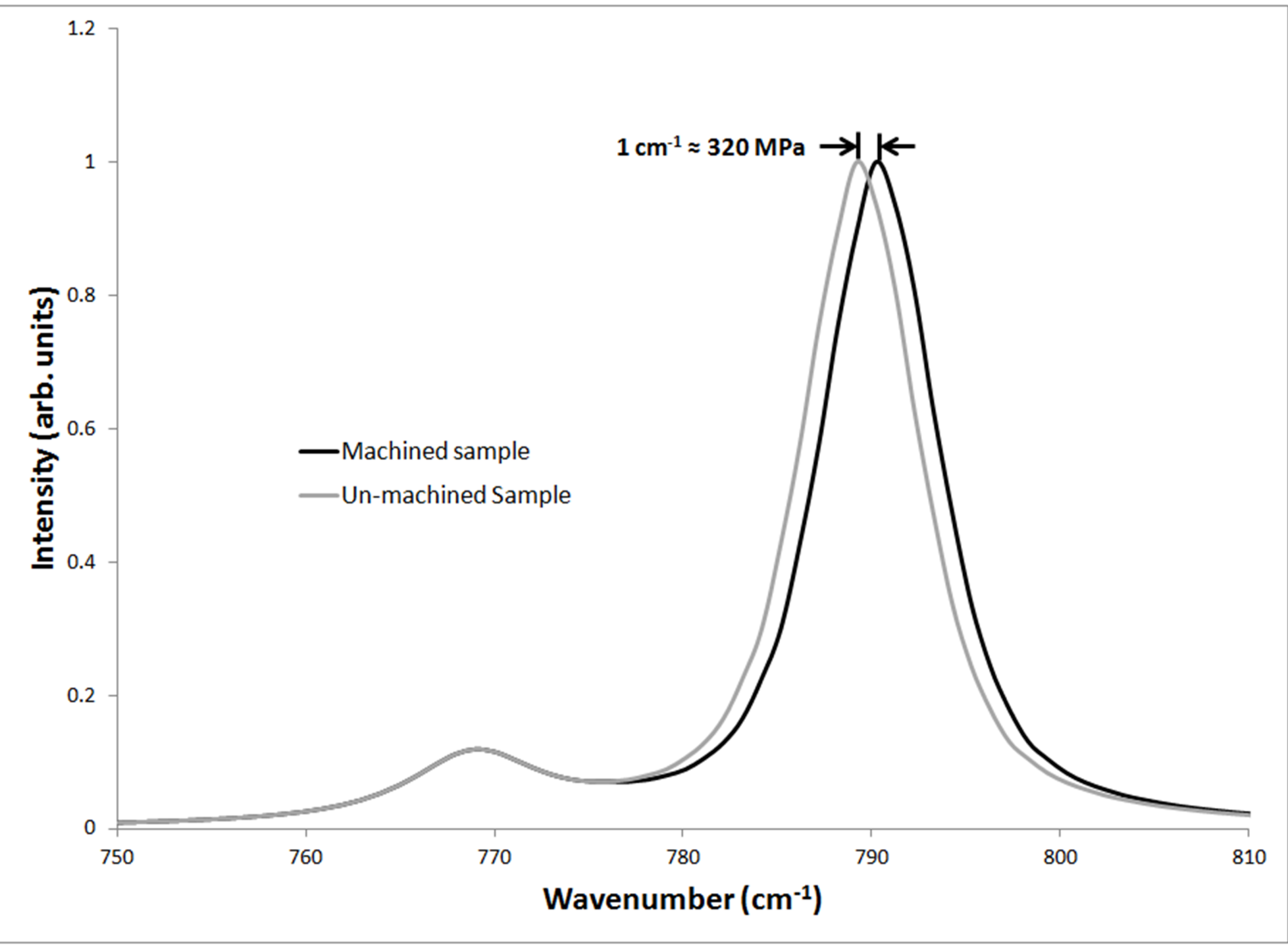


Figure 4

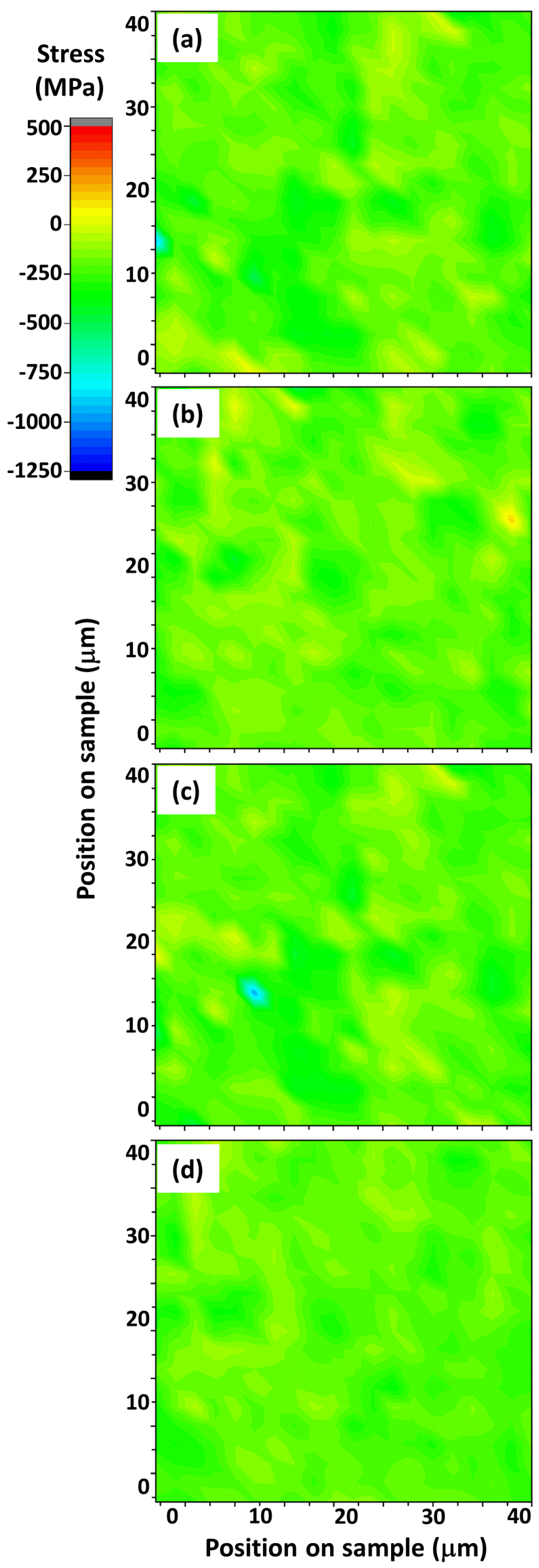


Figure 5
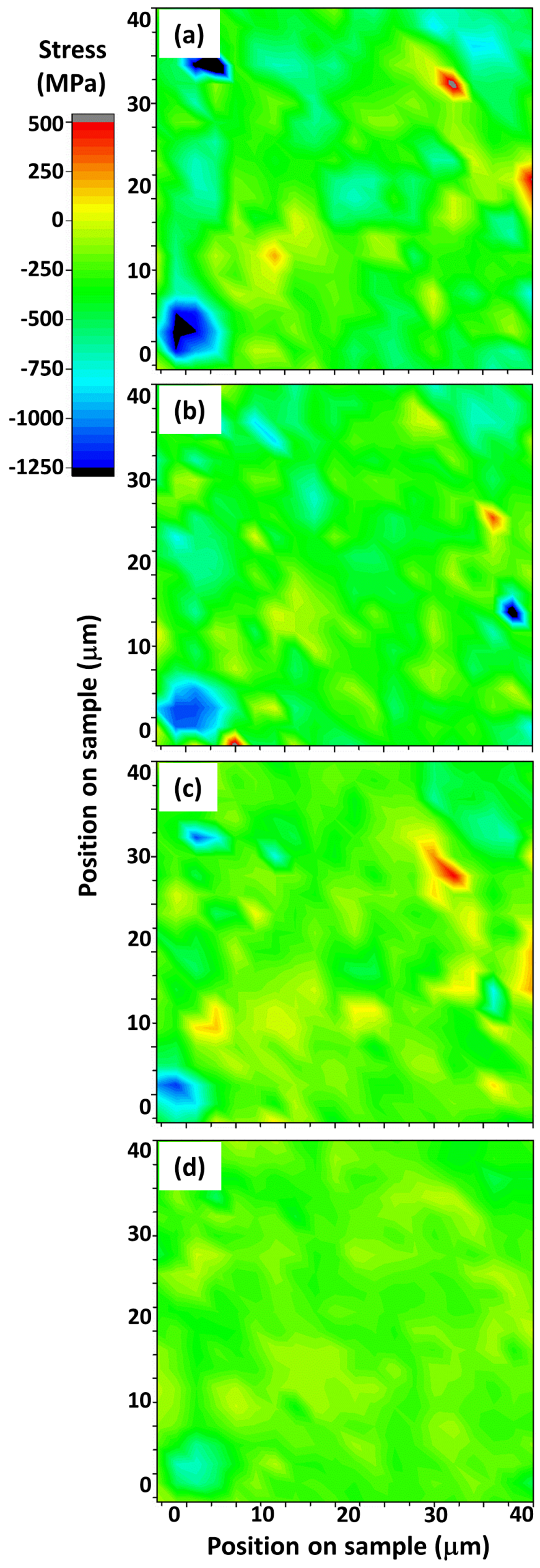
Figure 6
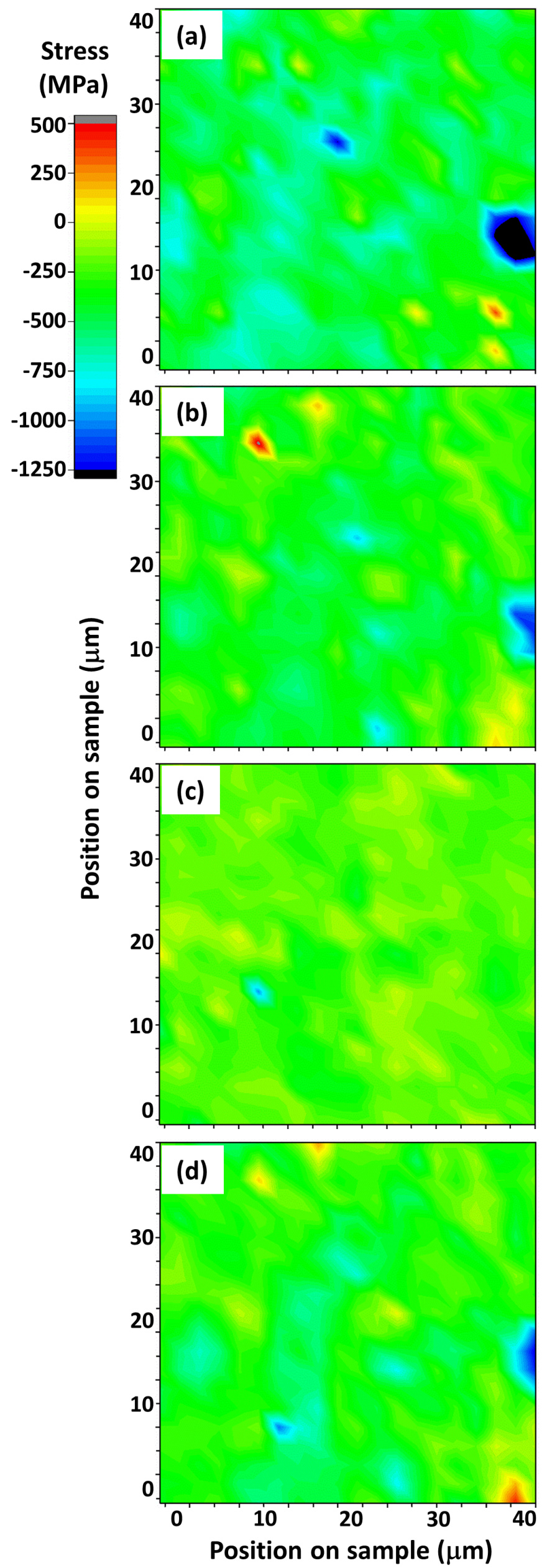
Figure 7

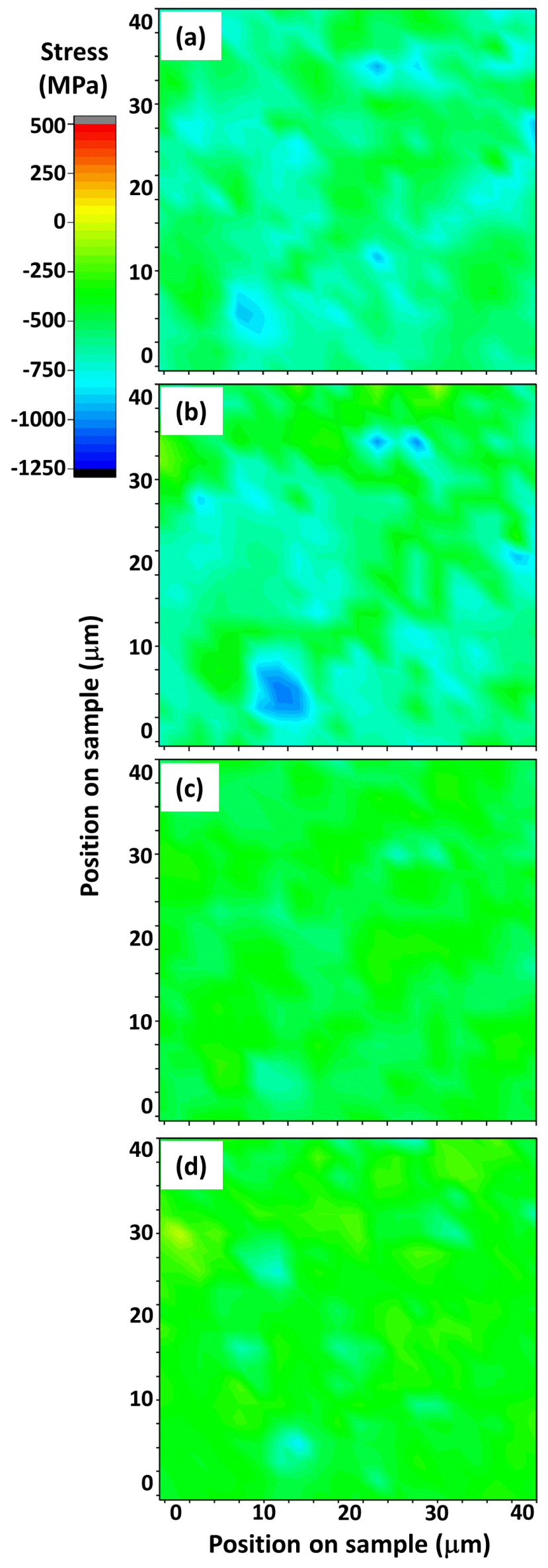


Figure 8

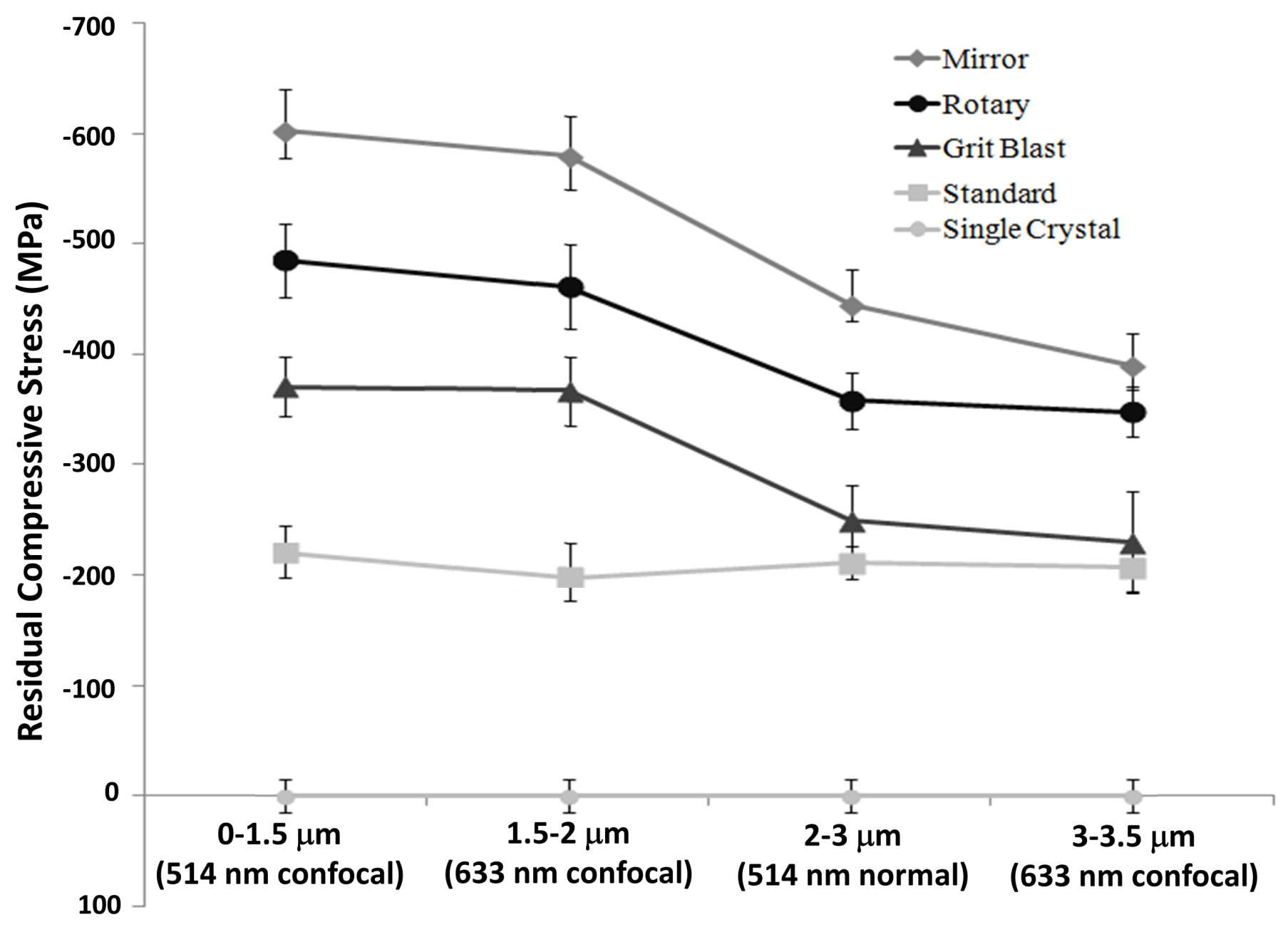

Approximate depth of penetration (Laser setting) 
Figure 9

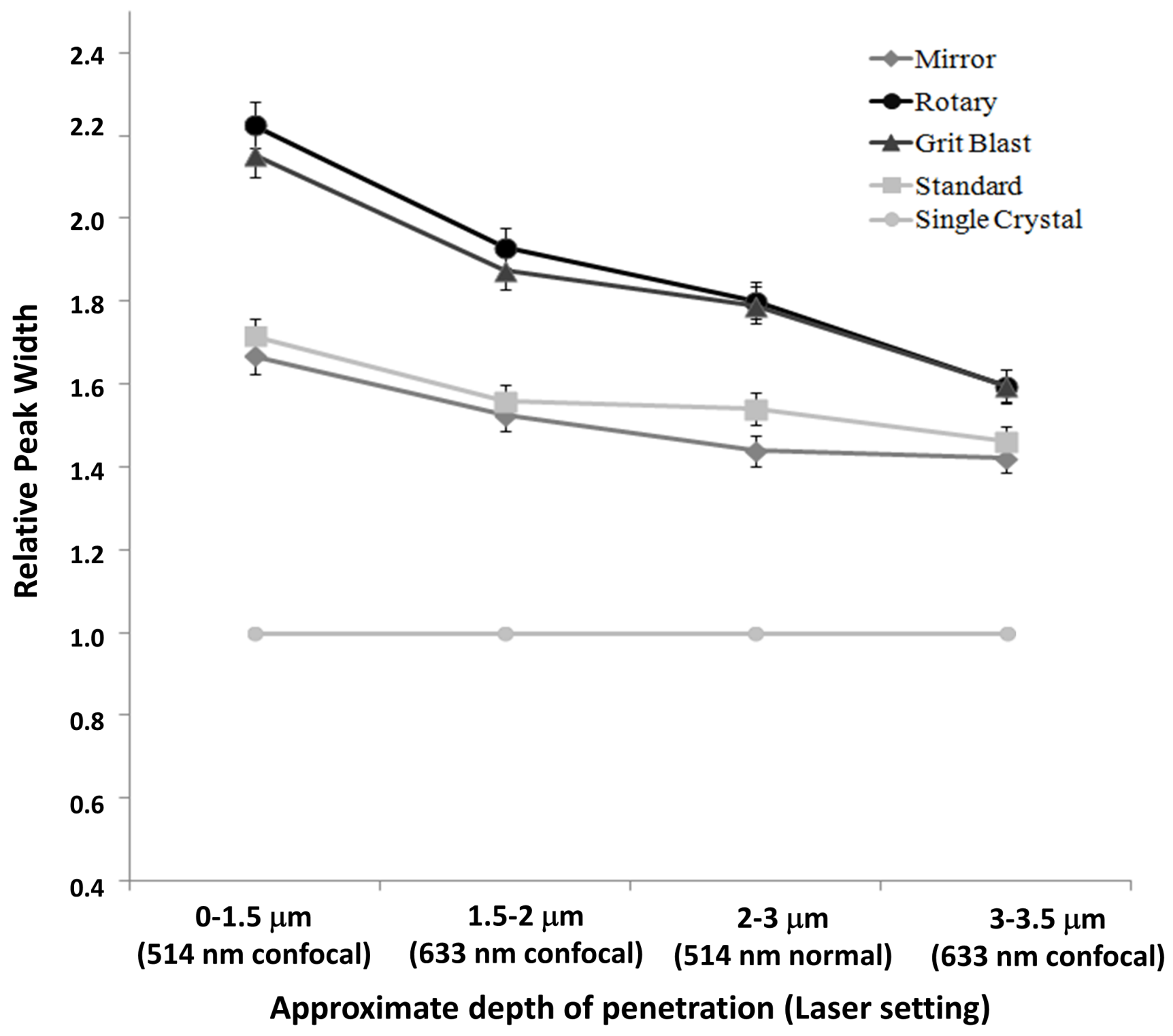


Figure 10

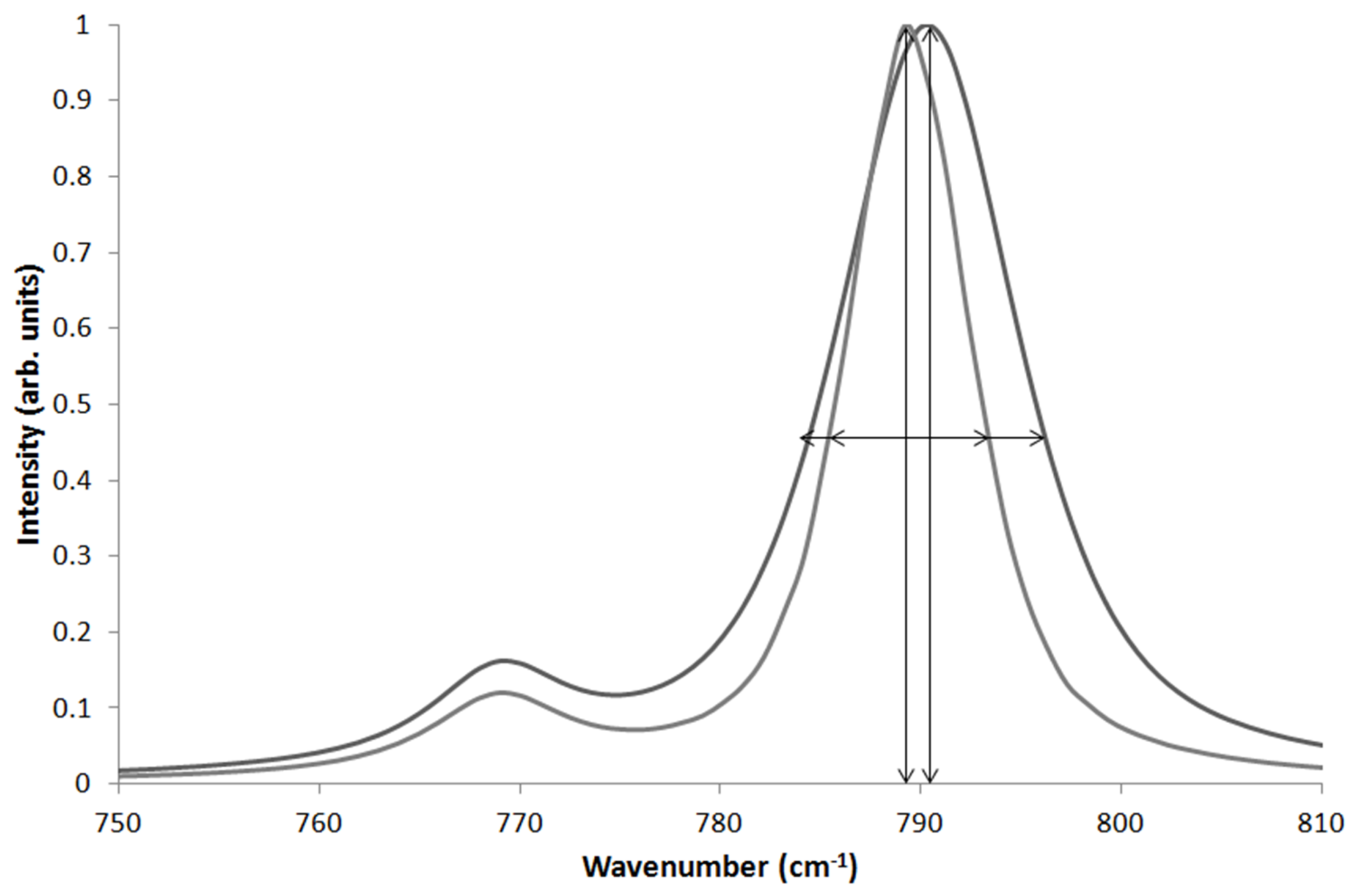

